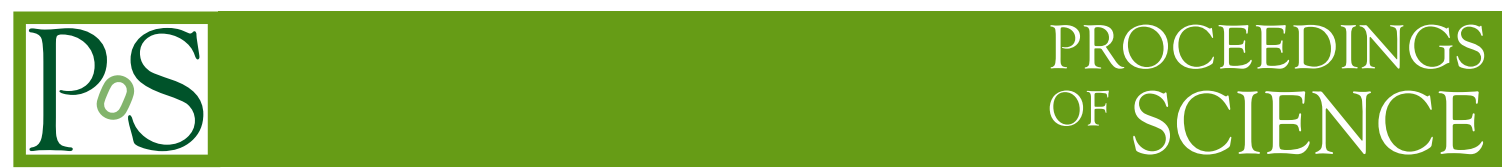

\title{
Heavy Flavor Spectroscopy and Production in CMS
}

\author{
Sara Fiorendi* \\ INFN and University of Milano Bicocca \\ on behalf of the CMS Collaboration \\ E-mail: sara.fiorendiecern.ch
}

\begin{abstract}
A review of recent results from the CMS experiment at the LHC in the Heavy Flavor sector is presented. We report on the measurement of the $\mathrm{X}(3872)$ production cross section, the observation of structures in the $J / \psi \phi$ spectrum, the observation of the $B_{c}$ meson and of a new $\Xi$ baryon and the measurement of beauty di-jet angular correlation.
\end{abstract}

14th International Conference on B-Physics at Hadron Machines April 8-12, 2013

Bologna, Italy

${ }^{*}$ Speaker. 


\section{Introduction}

The CMS experiment [1] at the Large Hadron Collider pursues a wide program in the Heavy Flavor physics sector. We present here some recent results of this program, namely, the measurement of the $\mathrm{X}(3872)$ production cross section and the observation of structures in the $J / \psi \phi$ spectrum. We also show the observation of a new $\Xi$ baryon and of the $B_{c}$ meson. Finally, we present a measurement of beauty di-jet angular correlation. Measurements are based on proton proton data collected at $\sqrt{s}=7 \mathrm{TeV}$.

\section{2. $\mathrm{X}(3872)$ production cross section}

The differential $\mathrm{X}(3872)$ production cross section is measured with $4.8 \mathrm{fb}^{-1}$ of data [2]. The cross section measurement proceeds by determining the ratio of the $\mathrm{X}(3872)$ and $\psi(2 \mathrm{~S})$ cross sections, where both states decay to $J / \psi \pi^{+} \pi^{-}$, with the subsequent decay of the $J / \psi$ into a muon pair. In the ratio evaluation, systematics uncertainties common to both states cancel.

The analysis is performed in the kinematic range of $p_{T}$ of the $J / \psi \pi^{+} \pi^{-}$system between 10 and $50 \mathrm{GeV}$ and rapidity within $|y|<1.2$. The corrections for detector acceptances and efficiencies are determined under the assumption that the $\mathrm{X}(3872)$ has quantum numbers $J^{P C}=1^{++}$and that both the $\mathrm{X}(3872)$ and the $\psi(2 \mathrm{~S})$ are unpolarized.

The events are selected at the trigger level identifying two opposite charge muons, fitted to a common vertex to produce $J / \psi$ candidates. Thresholds on dimuon momentum and rapidity are applied. The $J / \psi$ candidate is then combined with two opposite charge tracks, assumed to be pions. Additional requirements concern $\Delta R=\sqrt{(\Delta \eta)^{2}+(\Delta \phi)^{2}}(\Delta \eta$ and $\Delta \phi$ are the pseudorapidity and azimuthal angle differences between the pion and the $J / \psi$ candidate) and the Q value of the decay.

The ratio of the cross sections times the $J / \psi \pi^{+} \pi^{-}$branching fractions is obtained from the measured signal events for $\mathrm{X}(3872)$ and $\psi(2 \mathrm{~S})$ corrected for the efficiency and acceptance estimated from simulation. The cross section ratio as a function of the transverse momentum of the $J / \psi \pi^{+} \pi^{-}$system is shown in Fig. 1 .

The relative contribution to the total $\mathrm{X}(3872)$ yield resulting from $B$ hadron decays is also measured. Discrimination between promptly and non-promptly produced events is achieved by requiring a transverse decay length larger than $100 \mu \mathrm{m}$. Combining the non prompt fraction with the cross section ratio measurement and with a previous CMS result on the $\psi(2 \mathrm{~S})$ cross section [4], the prompt $\mathrm{X}(3872)$ production cross section is evaluated. Figure 1 shows the results compared to the predicted differential cross section for prompt X(3872) [5].

Finally, the measured dipion mass spectrum for $\mathrm{X}(3872) \rightarrow J / \psi \pi^{+} \pi^{-}$is studied and clearly favors the presence of an intermediate $\rho^{0}$ state.

\section{Observation of substructures in the $J / \psi \phi$ spectrum}

A study of the $J / \psi \phi$ mass spectrum from exclusive $B^{+} \rightarrow J / \psi \phi K^{+}$decays based on $5.2 \mathrm{fb}^{-1}$ of data is reported [6]. A candidate $J / \psi$ meson selected at the trigger level is combined with three charged tracks, assumed to be kaons, consistent with originating from the same $J / \psi$ vertex. The $\mathrm{K}^{+} \mathrm{K}^{-}$pair with the lower mass is considered as the $\phi$ candidate and must lie in the $\phi$ mass window. 

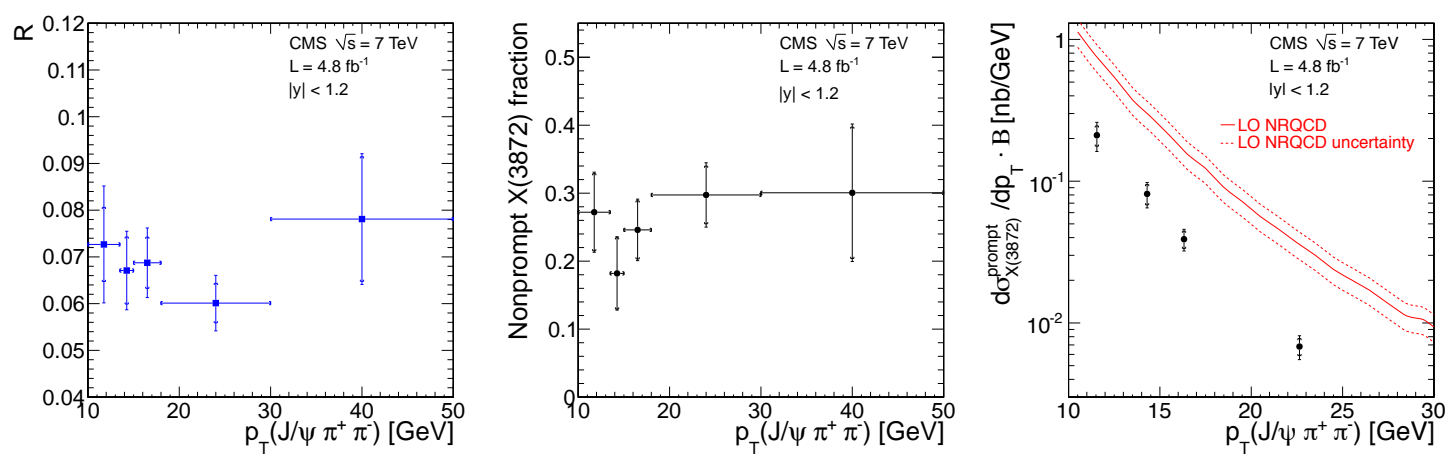

Figure 1: Ratios of the $\mathrm{X}(3872)$ and $\psi(2 \mathrm{~S})$ cross sections times branching fractions (left), $\mathrm{X}(3872)$ nonprompt fraction as a function of $p_{T}$ (center) and differential cross section for prompt $\mathrm{X}(3872)$ production times $\mathrm{BR}\left(\mathrm{X}(3872) \rightarrow J / \psi \pi^{+} \pi^{-}\right)$as a function of $p_{T}$ (right).

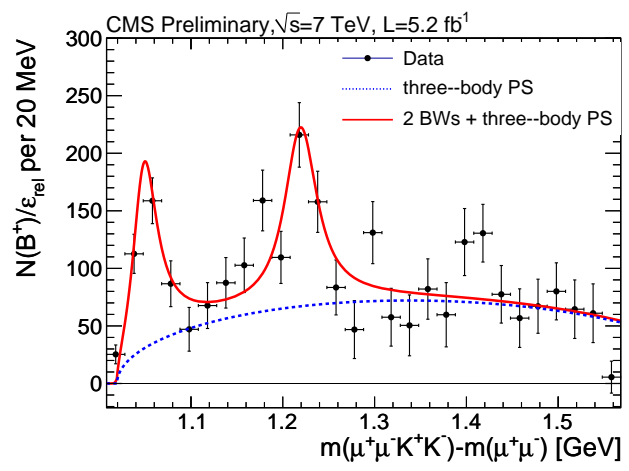

Figure 2: The relative efficiency corrected mass difference $\Delta m$ from the exclusive $B^{+} \rightarrow J / \psi \phi K^{+}$signal.

The $\Delta m=m\left(\mu^{+} \mu^{-} K^{+} K^{-}\right)-m\left(\mu^{+} \mu^{-}\right)$spectrum, corrected by the relative efficiency, is investigated. The observed structures are modeled by a $S$-wave relativistic Breit-Wigner function convoluted with a Gaussian resolution function, while the background is described by the three body phase space shape. Fig. 2 shows the $\Delta m$ spectrum and the fit of the data. The significance of the lower mass structure is greater than $5 \sigma$, while an evidence for a second structure in the same mass spectrum is seen.

\section{Observation of a new $\Xi_{b}$ baryon}

A new $b$ baryon is observed through its strong decay into $\Xi_{b}^{-} \pi^{+}$in $5.3 \mathrm{fb}^{-1}$ of data [7].

The known $\Xi_{b}^{-}$baryon is reconstructed via the decay chain $\Xi_{b}^{-} \rightarrow J / \psi \Xi^{-} \rightarrow \mu^{+} \mu^{-} \Lambda^{0} \pi^{-}$, with $\Lambda^{0} \rightarrow p \pi^{-}$. Once a sample of $\Xi_{b}^{-}$with a good signal-over-background ratio is selected, $\Xi_{b}^{-}$candidates within 2.5 standard deviations of the fitted peak value are combined with a track, assumed to be a pion, coming from the selected primary vertex and with charge opposite to that of the pion from the $\Xi^{-}$decay.

The $\Xi_{b}^{* 0}$ is searched in the $Q$ value domain, where $Q=M\left(J / \psi \Xi^{-} \pi^{+}\right)-M\left(J / \psi \Xi^{-}\right)-M\left(\pi^{+}\right)$. The background model is built using the measured momentum and angular distribution of $\Xi_{b}^{-}$ candidates and same sign pions. 


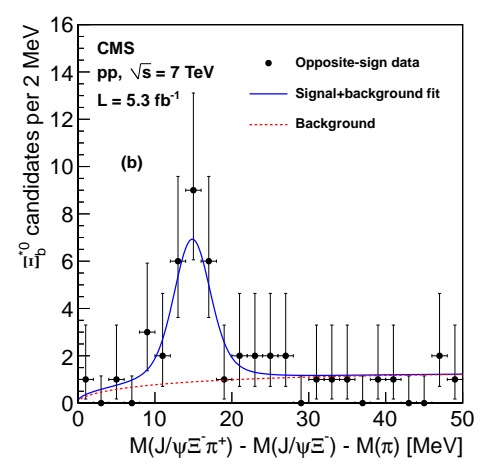

Figure 3: Opposite-sign Q distribution along with the result of the signal-plus-background fit (blue solid curve); the background term is also shown (red dashed curve).
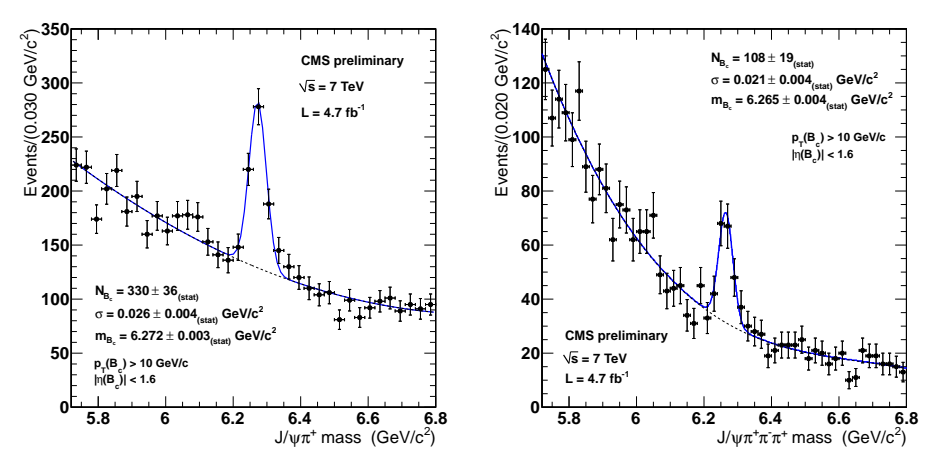

Figure 4: $B_{c}^{+} \rightarrow J / \psi \pi^{+}$(left) and $B_{c}^{+} \rightarrow J / \psi \pi^{+} \pi^{+} \pi^{-}$(right) signals.

The measured opposite-sign Q distribution is displayed in Fig. 3. A clear excess with respect to the expected background is observed and fitted with a Breit-Wigner distribution convoluted with a Gaussian function. The measured Q value is $14.84 \pm 0.74$ (stat) \pm 0.28 (syst) $\mathrm{MeV}$, resulting in a $b$-baryon mass of $5945.0 \pm 0.7$ (stat) \pm 0.3 (syst) \pm 2.7 (PDG) $\mathrm{MeV}$. The significance of the signal is evaluated to be $6.9 \sigma$.

\section{Observation of the $B_{c}$ meson}

We present the observation of the $B_{c}$ meson in two decay channels, $B_{c}^{+} \rightarrow J / \psi \pi^{+}$and $B_{c}^{+} \rightarrow$ $J / \psi \pi^{+} \pi^{+} \pi^{-}$in $4.7 \mathrm{fb}^{-1}$ of data [8]. The three pion decay mode has been observed for the first time by $\mathrm{LHCb}[9]$ and CMS has provided the only experimental confirmation so far.

The reconstruction strategy is driven by the $J / \psi$ identification at the trigger level through its decay into two muons. Either one or three charged tracks, assumed to be pions, are combined with the $J / \psi$ meson to form the $B_{c}$ candidate. A total yield of $330 \pm 36$ events is observed for the one pion decay channel, while $108 \pm 19$ events are observed for the three pion decay mode. The two signals are shown in Fig. 4. 


\section{Measurement of beauty di-jet angular correlation}

Angular correlations between pairs of beauty jets are studied in $3 \mathrm{pb}^{-1}$ of data [10]. Measurements of di-beauty jet production are presented as a function of the difference in azimuthal angle between the two b-tagged jets, $\Delta \phi$, and the combined separation variable $\Delta R=\sqrt{\Delta \phi^{2}+\Delta \eta^{2}}$.

Events are selected by a low-transverse-momentum single-muon trigger; $p_{T}(\mu)>8 \mathrm{GeV}$ and $\left|\eta^{\mu}\right|<2.1$ are required. Jets are reconstructed from "particle flow candidates" using the infraredand collinear-safe anti- $k_{t}$ jet clustering algorithm, and are required to have $p_{T}>30 \mathrm{GeV}$ and $|\eta|<2$.4. Only events with at least one mu-jet and one non-mu-jet are considered. The $b$-tagging is performed with the Track Counting algorithm [11][12]. Events with three or more jets passing the selections are vetoed.

The overall selection efficiency is determined with respect to the angular variables using the simulated event sample. The di-beauty signal in data is determined by a bin-by-bin "purity correction" to finally selected events. This correction is obtained by solving a system of four equations which relates the known b-tagging efficiencies to the fractional flavor content of the jet pair.

The differential beauty di-jet production cross section with respect to $\Delta \phi$ and $\Delta R$ is shown in Fig. 5. The total measured and theoretical cross sections for two beauty jets with $p_{T}>30 \mathrm{GeV}$ and $|\eta|<2.1$, with angular distance between them $\Delta R>0.6$, are also evaluated and summarized in Table 6.

Data are compared to theoretical predictions: PYTHIA shows disagreement in the low $\Delta \phi$ region while best describing the absolute normalization. CASCADE has regions of disagreement in both $\Delta \phi$ and $\Delta R$ and underestimates the absolute normalization of data. Finally, MADGRAPH gives the best account of the shape of the low angular region out of the three models considered while overestimating the absolute normalization.

\begin{tabular}{|c|c|c|c|}
\hline Data & PYTHIA & MADGRAPH & CASCADE \\
\hline $12.2 \pm 0.2$ (stat) ${ }_{-1.2}^{+1.6}$ (syst) & $13.18 \pm 0.02$ (stat) & $17.1 \pm 0.1$ (stat) & $9.48 \pm 0.04$ (stat) ${ }_{-2.65}^{+1.93}$ (syst) \\
\hline
\end{tabular}

Table 1: Total di-beauty jet measured and theoretical cross sections in nb

\section{Conclusions}

CMS is contributing to the enrichment of the Heavy Flavor physics, both in the production and decay sectors. The production cross section of the X(3872) and of pair of beauty jets have been measured. The $B_{c}$ meson has been observed in two decay modes and a new baryon $\left(\Xi_{b}^{* 0}\right)$ has been detected. Finally, the $J / \psi \phi$ mass spectrum shows some structures whose nature is still to be determined.

\section{References}

[1] S. Chatrchyan et al. (CMS Collaboration), The CMS experiment at the CERN LHC, JINST 3, S08004 (2008) 

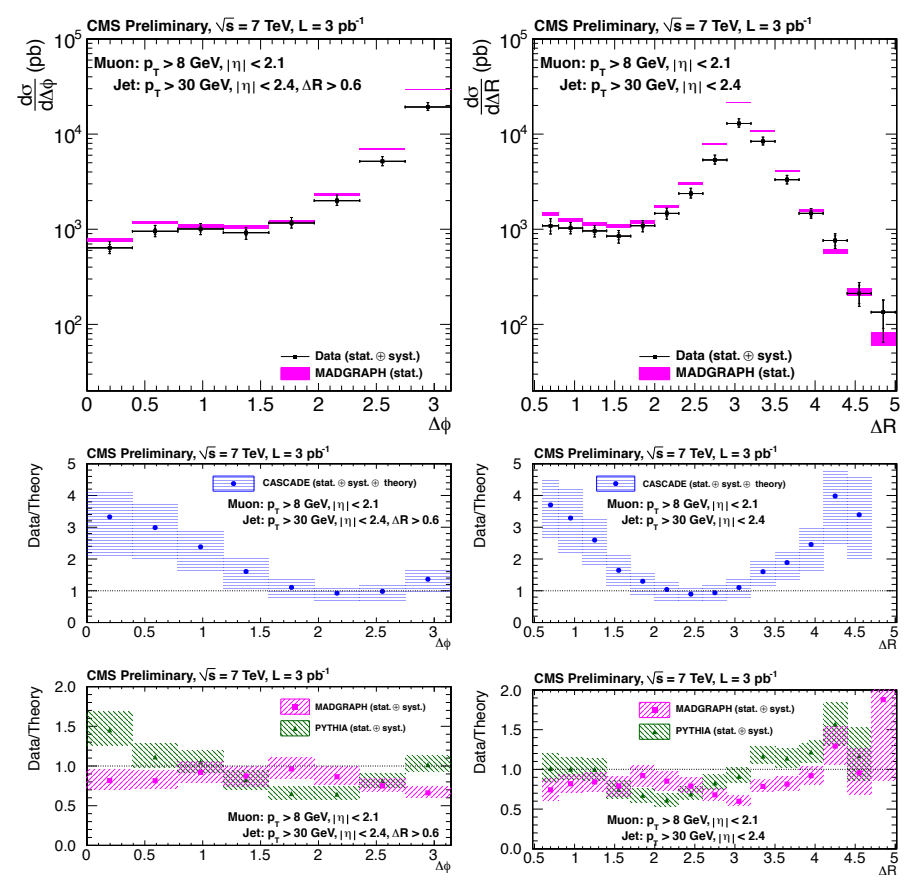

Figure 5: Differential di-beauty jet production cross section with respect to the $\Delta \phi$ (left) and $\Delta R$ (right) between two b-tagged jets.

[2] S. Chatrchyan et al. (CMS Collaboration), Measurement of the X(3872) production cross section via decays to $J / \psi \pi^{+} \pi^{-}$in pp collisions at $\sqrt{s}=7 \mathrm{TeV}$, JHEP 04 (2013) 154

[3] N. Brambilla et al., Heavy quarkonium: progress, puzzles, and opportunities Eur. Phys. J. C 71 (2011) 1534 [arXiv:1010.5827 [hep-ph]]

[4] S. Chatrchyan et al. (CMS Collaboration), $J / \psi$ and $\psi_{2 S}$ production in pp collisions at $\sqrt{s}=7 \mathrm{TeV}$, JHEP 1202 (2012) 011 [arXiv:1111.1557 [hep-ex]]

[5] P. Artoisenet and E. Braaten, Production of the X(3872) at the Tevatron and the LHC Phys. Rev. D 11 (2010) 114018

[6] S. Chatrchyan et al. (CMS Collaboration), CMS Physics Analysis Summary CMS-PAS-BPH-11-026

[7] S. Chatrchyan et al. (CMS Collaboration), Observation of a New $\Xi_{b}$ Baryon, Phys. Rev. Lett. 108 (2012) 252002

[8] S. Chatrchyan et al. (CMS Collaboration), Observation of $B_{c}$ decays to $J / \psi \pi$ and $J / \psi \pi \pi \pi$, CMS Physics Analysis Summary CMS-PAS-BPH-11-003 (2012)

[9] RAaij et al. (LHCb Collaboration), First observation of the decay $B_{c}^{+} \rightarrow J / \psi \pi^{+} \pi^{-} \pi^{+}$, Phys. Rev. Lett. 108 (2012) 251802 [arXiv:1204.0079 [hep-ex]]

[10] S. Chatrchyan et al. (CMS Collaboration), Measurement of Angular Correlations Between Beauty Jets Produced in pp Collisions at 7 TeV, CMS Physics Analysis Summary CMS-PAS-BPH-10-019 (2013)

[11] S. Chatrchyan et al. (CMS Collaboration), Commissioning of b-jet identification with pp collisions at $\sqrt{(s)}=7 \mathrm{TeV}$, CMS Physics Analysis Summary CMS-PAS-BTV-10-001 (2010)

[12] S. Chatrchyan et al. (CMS Collaboration), Performance of b-jet identification in CMS, CMS Physics Analysis Summary CMS-PAS-BTV-11-001 (2011) 